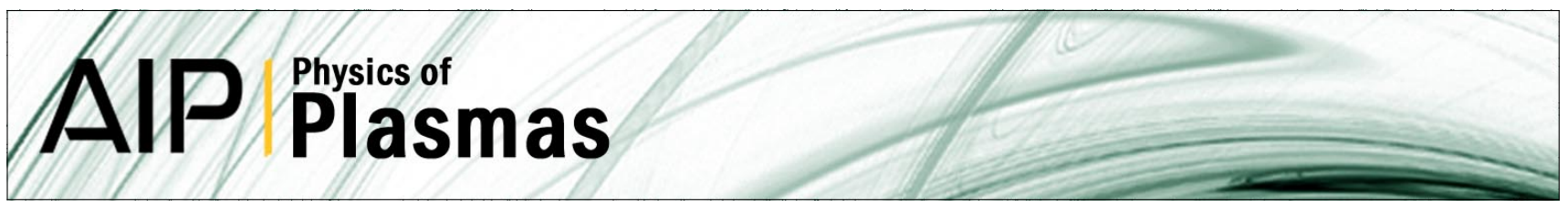

\title{
Boundary conditions for plasma fluid models at the magnetic presheath entrance
}

J. Loizu, P. Ricci, F. D. Halpern, and S. Jolliet

Citation: Phys. Plasmas 19, 122307 (2012); doi: 10.1063/1.4771573

View online: http://dx.doi.org/10.1063/1.4771573

View Table of Contents: http://pop.aip.org/resource/1/PHPAEN/v19/i12

Published by the American Institute of Physics.

\section{Related Articles}

Experimental investigation of virtual cathode sheath in the presence of secondary electrons produced by streaming ions

Phys. Plasmas 19, 113511 (2012)

Valid flow combinations for stable sheath in a magnetized multiple ion species plasma

Phys. Plasmas 19, 113507 (2012)

Properties of a warm plasma collisional sheath in an oblique magnetic field

Phys. Plasmas 19, 113504 (2012)

Investigation of the sheath formation in a dusty plasma containing energetic electrons and nano-size dust grains Phys. Plasmas 19, 103505 (2012)

Potential contour shaping and sheath behavior with wall electrodes and near-wall magnetic fields in Hall thrusters Phys. Plasmas 19, 103502 (2012)

\section{Additional information on Phys. Plasmas}

Journal Homepage: http://pop.aip.org/

Journal Information: http://pop.aip.org/about/about_the_journal

Top downloads: http://pop.aip.org/features/most_downloaded

Information for Authors: http://pop.aip.org/authors

\section{ADVERTISEMENT}

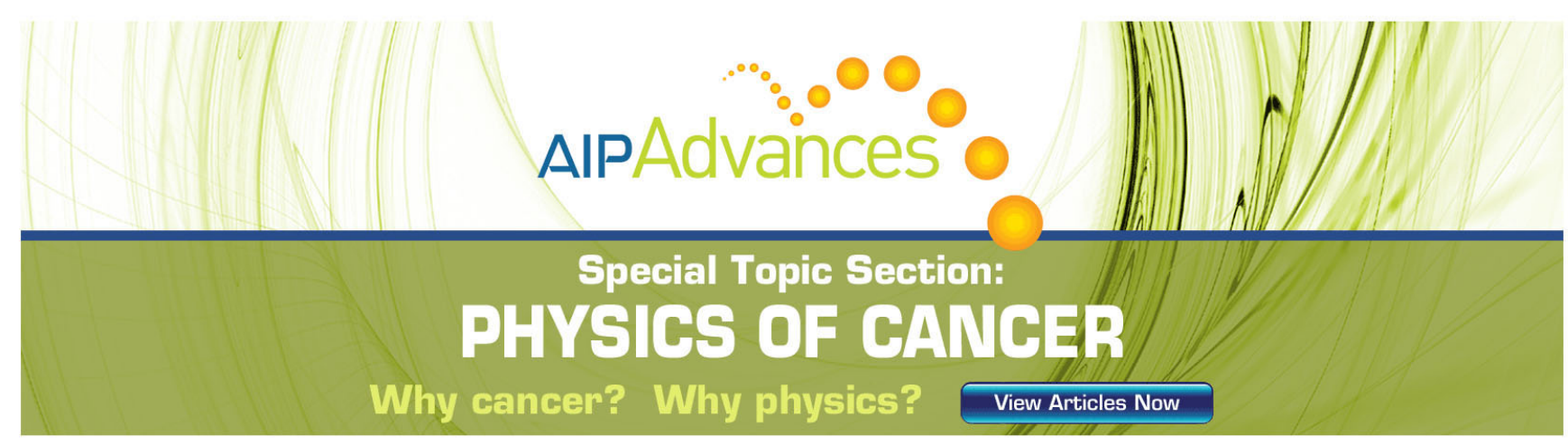




\title{
Boundary conditions for plasma fluid models at the magnetic presheath entrance
}

\author{
J. Loizu, P. Ricci, F. D. Halpern, and S. Jolliet ${ }^{a)}$ \\ Centre de Recherches en Physique des Plasmas-École Polytechnique Fédérale de Lausanne, \\ Association EURATOM-Confédération Suisse, CH-1015 Lausanne, Switzerland
}

(Received 7 October 2012; accepted 20 November 2012; published online 13 December 2012)

The proper boundary conditions at the magnetic presheath entrance for plasma fluid turbulence models based on the drift approximation are derived, focusing on a weakly collisional plasma sheath with $T_{i} \ll T_{e}$ and a magnetic field oblique to a totally absorbing wall. First, the location of the magnetic presheath entrance is rigorously derived. Then boundary conditions at the magnetic presheath entrance are analytically deduced for $v_{\| i}, v_{\| e}, n, \phi, T_{e}$, and for the vorticity $\omega=\nabla_{\perp}^{2} \phi$. The effects of $\mathrm{E} \times \mathrm{B}$ and diamagnetic drifts on the boundary conditions are also investigated. Kinetic simulations are performed that confirm the analytical results. Finally, the new set of boundary conditions is implemented in a three-dimensional global fluid code for the simulation of plasma turbulence and, as an example, the results of a tokamak scrape-off layer simulation are discussed. The framework presented can be generalized to obtain boundary conditions at the magnetic presheath entrance in more complex scenarios. [http://dx.doi.org/10.1063/1.4771573]

\section{INTRODUCTION}

The magnetized plasma-wall transition determines the boundary conditions for all laboratory magnetized plasmas, imposing the plasma losses at the wall and therefore the steady state profiles and the plasma circulation. When the magnetic field is oblique with respect to an absorbing wall, the plasma-wall transition consists of three subregions (see, e.g., Ref. 1 for a review): the collisional presheath (CP), the magnetic presheath (MP), also called Chodura sheath, and the Debye sheath (DS), which is in contact with the wall. In each of these regions, a potential drop proportional to the electron temperature is observed, $\Delta \phi \propto T_{e}$, but on very different spatial scales. The CP width typically scales with the ion mean free path, $\lambda_{m f}$. The scale length of the MP is the ion sound larmor radius, $\rho_{s}$. The DS width has a scale length of the order of the Debye length, $\lambda_{D}$. In the CP plasma is quasineutral, ions are magnetized and accelerated towards the wall, reaching the plasma sound speed $c_{s}=\sqrt{T_{e} / m_{i}}$ at the MP entrance along the magnetic field direction. The MP is also quasineutral but the electric field is strong enough to demagnetize the ions, which are deflected and reach the DS entrance flowing at $c_{s}$ in the direction normal to the wall. Inside the DS quasineutrality is violated.

Plasma turbulence fluid codes (see Refs. 2-8 for some examples) are based on the quasineutrality approximation, which breaks down at the DS entrance. They are also typically based on the ion drift approximation (IDA), which breaks down in the MP. Therefore, the magnetic presheath cannot be described by a fluid model based on the IDA. More precisely, in plasma fluid turbulence codes the analysis of the dynamics is usually split into the direction parallel and perpendicular to the magnetic field, i.e., by decomposing

\footnotetext{
${ }^{a)}$ Electronic address: joaquim.loizu@epfl.ch.
}

$\mathbf{v}_{i}=v_{\| i} \mathbf{b}+\mathbf{v}_{\perp i}$, where $\mathbf{b}=\mathbf{B} / B$. The drift ordering usually adopted, $d / d t \ll \omega_{c i}$, where $d / d t=\partial_{t}+\mathbf{v}_{i} \cdot \nabla$, implies that the inertia term is small compared to the electric and magnetic forces in the ion momentum equation, which in the cold ion limit is

$$
m_{i} n \frac{d}{d t} \mathbf{v}_{i}=e n \mathbf{E}+e n \mathbf{v}_{i} \times \mathbf{B} .
$$

One can therefore write the perpendicular velocity as $\mathbf{v}_{\perp i}=\mathbf{v}_{E}+\mathbf{v}_{p o l}$, where $\mathbf{v}_{E}=\mathbf{E} \times \mathbf{B} / B^{2}$ is the leading order term, and $\mathbf{v}_{p o l}=\left(\mathbf{b} / \omega_{c i}\right) \times d \mathbf{v}_{\perp i} / d t$ is the polarization drift velocity which contains all terms of order one and higher in $\left(1 / \omega_{c i}\right) d / d t$. Within the IDA, only the first order terms are retained, leading to

$$
\mathbf{v}_{\perp i}=\mathbf{v}_{E}+\frac{\mathbf{b}}{\omega_{c i}} \times \frac{d^{0}}{d t} \mathbf{v}_{E}
$$

where $d_{t}^{0}=\partial_{t}+\left(v_{\| i} \mathbf{b}+\mathbf{v}_{E}\right) \cdot \nabla$. In the MP, the deflection of the sonic ion flow from the direction parallel to the magnetic field to the direction of the electric field, which is normal to the wall, requires that ions are demagnetized and therefore violates the IDA. In fact, in the MP the ion inertia term is comparable to the other terms in Eq. (1), $m_{i} n\left(\mathbf{v}_{i} \cdot \nabla\right) \mathbf{v}_{\perp i} \sim e n \mathbf{v}_{\perp i} \times \mathbf{B}$, which provides a scaling for the size of the MP, $\lambda_{m}$, since $m_{i} c_{s}^{2} / \lambda_{m} \sim e c_{s} B$, and hence $\lambda_{m} \sim c_{s} / \omega_{c i}=\rho_{s}$. Thus, in the MP where the electric field varies on a scale length of the order of the ion sound Larmor radius, the ion motion cannot be described within the ion drift approximation. As a consequence, plasma turbulence fluid codes based on the IDA require boundary conditions at the MP entrance in order to account correctly for the plasmawall transition.

The magnetic presheath has been studied since the pioneering work of Chodura, ${ }^{9}$ followed by an extensive research 
effort that has brought to light many important aspects of this physical system, such as the effect of collisions, ${ }^{10-12}$ magnetic field angle, ${ }^{9,12-14} \mathbf{E} \times \mathbf{B}$ and diamagnetic drifts, ${ }^{15-20}$ and finite ion temperature..$^{9,21}$ Most of these studies provide a boundary condition for the parallel ion velocity at the MP entrance, whereas the boundary conditions for the other fluid quantities remain unclear.

The goal of the present article is to provide a complete set of boundary conditions to be used at the MP entrance in IDA-based fluid codes. We target a set of boundary conditions which can faithfully supply the sheath physics to the fluid codes and which, at the same time, remain simple enough to be easily implemented. We work under the assumption of a weakly collisional, steady-state plasma sheath with cold ions and in contact with a totally absorbing wall, and we assume that gradients in the directions parallel to the wall are on a scale much larger than $\rho_{s}$. In this framework, we determine rigorously the MP entrance condition and the boundary conditions for the plasma density and temperature, the electrostatic potential, the ion and electron parallel velocities, and the vorticity. The correctness of these boundary conditions is verified via kinetic simulations of the magnetized plasma-wall transition and the new set of boundary conditions is then implemented in GBS (Global Braginskii Solver), a threedimensional global fluid code based on the IDA that has been used to simulate plasma turbulence in basic plasma physics experiments and in the tokamak scrape-off layer (SOL). ${ }^{6} \mathrm{We}$ remark that the framework presented herein can be generalized to obtain boundary conditions at the magnetic presheath entrance in more complex scenarios.

The present paper is organized as follows. In Sec. II, we derive the condition that defines the MP entrance. Boundary conditions at this location are derived in Sec. III for the parallel ion and electron velocities, the plasma potential, the density, the electron temperature, and the vorticity. In Sec. IV, results from kinetic simulations are presented in order to validate the analytical results of Secs. II and III. The new set of boundary conditions is implemented in GBS. Details of the implementation and an example of a simulation are presented in Sec. V. The conclusions follow in Sec. VI. In the Appendix we present the derivation of the MP entrance condition when the assumption of isothermal electrons is relaxed.

\section{DERIVATION OF THE MAGNETIC PRESHEATH ENTRANCE CONDITION}

We consider a weakly collisional, steady-state plasma in contact with an absorbing wall, with $T_{i} \ll T_{e}$ and for which $\lambda_{D} \ll \rho_{s} \ll \lambda_{m f p}$. We assume a constant magnetic field oblique to the wall at an angle $\alpha$. For a suitable analytical description we use a field aligned coordinate system, $(x, y$, $z$ ), where $z$ is along $\mathbf{B}, x$ is perpendicular to $\mathbf{B}$ and parallel to the wall, and $y$ is perpendicular to both $x$ and $z$, directed towards the wall (see Fig. 1). In such geometry the magnetic field is $\mathbf{B}=\left(0,0, B_{0}\right)$. We also define the coordinate normal to the wall surface, $s=y \cos \alpha+z \sin \alpha$.

We consider the presence of plasma gradients in the $x$ direction with an ordering $\epsilon=\rho_{s} / L_{n} \sim \rho_{s} / L_{\phi} \sim \rho_{s} / L_{T_{e}} \ll 1$,
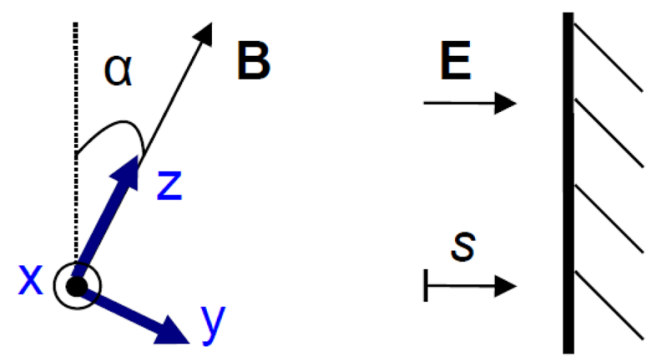

FIG. 1. Schematic representation of the sheath geometry. The magnetic field $\mathbf{B}$ is oblique to the wall at an angle $\alpha$. The wall is indicated on the right together with the sheath electric field $\mathbf{E}$, which is along the normal direction $s$.

where $L_{n}, L_{\phi}$, and $L_{T_{e}}$ are the density, potential, and temperature scale lengths in the $x$ direction. Since the MP electric field has a characteristic scale length of the order of $\rho_{s}$, it is much stronger than the electric field present in the bulk plasma, and gradients eventually dominate along the $s$ direction. We remark that plasma gradients in the direction perpendicular to both $s$ and $x$ do not affect the results derived herein, therefore we do not consider them for the sake of simplicity.

Space and time are expressed in the natural units of the system, using a reference electron temperature $T_{e 0}$. Namely, the electron temperature and the electrostatic potential are normalized as $T_{e} \rightarrow T_{e} / T_{e 0}$ and $\phi \rightarrow e \phi / T_{e 0}$, while space and time are normalized as $x \rightarrow x / \rho_{s 0}$ and $t \rightarrow \omega_{c i} t$, where $\rho_{s 0}=c_{s 0} / \omega_{c i}, c_{s 0}=\sqrt{T_{e 0} / m_{i}}$, and $\omega_{c i}=e B_{0} / m_{i}$. Velocities are therefore normalized to the sound speed, $v \rightarrow v / c_{s 0}$. Finally, the density is normalized to a reference density, $n \rightarrow n / n_{0}$. In the following, all quantities will be expressed in normalized units.

In order to describe the steady-state dynamics of the CP, we now write a system of equations including the ion continuity equation and the ion and electron parallel momentum equations. We then derive the condition defining the MP entrance by following an approach similar to that described in Ref. 22 in the case of a magnetic field normal to the wall.

The steady-state continuity equation for ions is $\nabla \cdot\left(n \mathbf{v}_{i}\right)=S_{p i}$, where $S_{p i}$ is the ion particle source. Using the relation $v_{s i}=v_{\| i} \sin \alpha+v_{y i} \cos \alpha$, it can be written as

$$
v_{s i} \partial_{s} n+n \sin \alpha \partial_{s} v_{\| i}+n \cos \alpha \partial_{s} v_{y i}+n \partial_{x} v_{x i}+v_{x i} \partial_{x} n=S_{p i}
$$

Equation (3) can be simplified by noting that the ion drift approximation, Eq. (2), can be used in the CP to express the perpendicular velocities. At the zeroth order in $\left(1 / \omega_{c i}\right) d / d t$, namely, neglecting the ion polarization drift, Eq. (2) gives $v_{x i}=-\cos \alpha \partial_{s} \phi$ and $v_{y i}=\partial_{x} \phi$. As a matter of fact, these expressions describe well the perpendicular ion velocities in the $\mathrm{CP}$, as shown later in the kinetic simulation results (see Sec. IV). Therefore the third and fourth terms in Eq. (3) cancel each other since we have $n \cos \alpha \partial_{s} v_{y i}$ $=n \cos \alpha \partial_{s} \partial_{x} \phi=-n \partial_{x} v_{x i}$, and for the fifth term we have $v_{x i} \partial_{x} n=-\partial_{x} n \cos \alpha \partial_{s} \phi \sim O(\epsilon)$. The continuity equation, Eq. (3), can then be rewritten in a simpler form, that is

$$
v_{s i} \partial_{s} n+n \sin \alpha \partial_{s} v_{\| i}-\partial_{x} n \cos \alpha \partial_{s} \phi=S_{p i},
$$


which is valid in the $\mathrm{CP}$ up to the MP entrance, where the polarization drift becomes important and the IDA breaks down.

We now consider the parallel component of the ion momentum equation, Eq. (1), which in steady-state is

$$
n\left(v_{s i} \partial_{s}+v_{x i} \partial_{x}\right) v_{\| i}=-n \sin \alpha \partial_{s} \phi+S_{\| m i},
$$

where we have introduced a source of momentum, $S_{\| m i}$, eventually present in the system and due to either injection of particles, ionization or collisions. Using again the relation $v_{x i}=-\cos \alpha \partial_{s} \phi$, Eq. (5) can be written as

$$
n v_{s i} \partial_{s} v_{\| i}+n\left(\sin \alpha-\partial_{x} v_{\| i} \cos \alpha\right) \partial_{s} \phi=S_{\| m i} \text {. }
$$

Finally, we consider the steady-state momentum equation for electrons, that is

$$
n\left(\mathbf{v}_{e} \cdot \nabla\right) \mathbf{v}_{e}=-\mu\left(n \mathbf{E}+n \mathbf{v}_{\mathbf{e}} \times \mathbf{b}+\nabla p_{e}\right)+\mathbf{S}_{m e},
$$

where $\mu=m_{i} / m_{e}$ and $p_{e}=n T_{e}$. Equation (7) can be simplified since $\mu \gg 1$, and therefore the electron inertia term can be neglected almost all the way up to the wall (electron inertia may become important only if the electric field varies on a scale length that is comparable to the electron gyroradius $\rho_{e}$ ). Moreover, it is reasonable to assume isothermal electrons in the $\mathrm{CP}$, namely, $\partial_{s} T_{e}=0$; this considerably simplifies the calculation and the expressions of the boundary conditions (a complete calculation relaxing the hypothesis $\partial_{s} T_{e}=0$ is presented in the Appendix, which shows that the temperature gradient is in fact small at the MP entrance). Hence, we have $\partial_{s} p_{e}=T_{e} \partial_{s} n$, and the parallel component of Eq. (7) is

$$
\mu \sin \alpha T_{e} \partial_{s} n-\mu \sin \alpha n \partial_{s} \phi=S_{\| m e} .
$$

The ion continuity equation, Eq. (4), and the parallel ion and electron momentum equations, Eqs. (6) and (8), form a system of equations,

$$
\begin{gathered}
v_{s i} \partial_{s} n+n \sin \alpha \partial_{s} v_{\| i}-\partial_{x} n \cos \alpha \partial_{s} \phi=S_{p i}, \\
n v_{s i} \partial_{s} v_{\| i}+n\left(\sin \alpha-\partial_{x} v_{\| i} \cos \alpha\right) \partial_{s} \phi=S_{\| m i}, \\
\mu \sin \alpha T_{e} \partial_{s} n-\mu \sin \alpha n \partial_{s} \phi=S_{\| m e},
\end{gathered}
$$

containing three unknowns $\left(n, v_{\| i}, \phi\right)$ and their respective gradients. The system of equations (9) can also be written as a matrix system $\mathbf{M} \vec{X}=\vec{S}$, where

$$
\vec{X}=\left(\begin{array}{c}
\partial_{s} n \\
\partial_{s} v_{\| i} \\
\partial_{s} \phi
\end{array}\right), \quad \vec{S}=\left(\begin{array}{c}
S_{p i} \\
S_{\| m i} \\
S_{\| m e}
\end{array}\right)
$$

and

$$
\mathbf{M}=\left(\begin{array}{ccc}
v_{s i} & n \sin \alpha & -\partial_{x} n \cos \alpha \\
0 & n v_{s i} & n\left(\sin \alpha-\partial_{x} v_{\| i} \cos \alpha\right) \\
\mu \sin \alpha T_{e} & 0 & -\mu n \sin \alpha
\end{array}\right) .
$$

The system of equations (9) is valid in the CP up to the MP entrance, where the IDA breaks down. In the CP, gra- dients are small and due to the presence of the sources. At the MP entrance, gradients become large, $\partial_{s} \sim 1$, and the source terms are much smaller than any other term in the fluid equations, i.e. $\left|M_{i j} X_{j}\right| \gg\left|S_{i}\right|$ for all $i, j$ such that $M_{i j} \neq 0$. In other words, nonzero gradients can be sustained without sources at the MP entrance, which leads to $\mathbf{M} \vec{X} \simeq 0$ at this location. Now, the presence of nonzero gradients imposes $\operatorname{det}(\mathbf{M})=0$, which defines the position of the MP entrance. This condition can be written as

$$
v_{s i}=c_{s} \sin \alpha\left(\frac{c_{s}}{2 \tan \alpha} \frac{\partial_{x} n}{n} \pm \sqrt{1+\left(\frac{c_{s}}{2 \tan \alpha} \frac{\partial_{x} n}{n}\right)^{2}-\frac{\partial_{x} v_{\| i}}{\tan \alpha}}\right)
$$

Notice that there are two solutions corresponding to the two opposite ends of the field line; we keep the positive solution for which the coordinate $s$ increases moving towards the wall, as in Fig. 1. Recalling that $v_{s i}=v_{\| i} \sin \alpha+v_{y i} \cos \alpha$ and that $v_{y i}=\partial_{x} \phi \sim O(\epsilon)$, we can deduce that $\partial_{x} v_{\| i} \sin \alpha=\partial_{x} v_{s i}$ $+O\left(\epsilon^{2}\right)$. Therefore, from Eq. (12) we have that $\partial_{x} v_{\| i}$ $=\partial_{x} c_{s}+O\left(\epsilon^{2}\right)$, with $\partial_{x} c_{s}=\partial_{x} T_{e} /\left(2 \sqrt{T_{e}}\right)$. We can thus write Eq. (12) as

$$
v_{s i}=c_{s} \sin \alpha\left(\theta_{n}+\sqrt{1+\theta_{n}^{2}-\theta_{T_{e}}}\right),
$$

where

$$
\begin{aligned}
\theta_{n} & =\frac{c_{s}}{2 \tan \alpha} \frac{\partial_{x} n}{n}, \\
\theta_{T_{e}} & =\frac{c_{s}}{2 \tan \alpha} \frac{\partial_{x} T_{e}}{T_{e}} .
\end{aligned}
$$

Retaining only first order terms in $\theta_{n} \sim \theta_{T_{e}} \sim \epsilon / \tan \alpha$, we obtain

$$
v_{s i}=c_{s} \sin \alpha\left(1+\theta_{n}-\theta_{T_{e}} / 2\right) .
$$

\section{BOUNDARY CONDITIONS AT THE MAGNETIC PRESHEATH ENTRANCE}

We now derive the boundary conditions for fluid turbulence codes at the MP entrance for the parallel ion and electron velocities, the plasma density, the electron temperature, the electrostatic potential, and the vorticity.

\section{A. Parallel ion velocity}

Recalling that $v_{s i}=v_{y i} \cos \alpha+v_{\| i} \sin \alpha$ and $v_{y i}=\partial_{x} \phi$, the parallel ion velocity at the MP entrance can be obtained by using Eq. (16). This leads to

$$
\begin{aligned}
v_{\| i} & =c_{s}\left(1+\theta_{n}-\theta_{T_{e}} / 2\right)-\partial_{x} \phi / \tan \alpha \\
& =c_{s}\left(1+\theta_{n}-\frac{1}{2} \theta_{T_{e}}-\frac{2 \phi}{T_{e}} \theta_{\phi}\right),
\end{aligned}
$$

where 


$$
\theta_{\phi}=\frac{c_{s}}{2 \tan \alpha} \frac{\partial_{x} \phi}{\phi}
$$

In the limit $\theta_{n}=\theta_{T_{e}}=\theta_{\phi}=0$, Eq. (17) retrieves the socalled Bohm-Chodura criterion $v_{\| i}=c_{s}{ }^{25}$ In the presence of plasma gradients in the $x$ direction, the main correction in Eq. (17) is typically due to the potential gradient. In fact, assuming $\theta_{n} \sim \theta_{T_{e}} \sim \theta_{\phi}$ and $\phi \approx 3 T_{e}$, the correction related with the potential gradient is six times larger than the density gradient correction, and twelve times larger than the temperature gradient one. We note that the correction given by the $\mathbf{E} \times \mathbf{B}$ drift, namely, the last term in Eq. (17), is valid at any order in $\epsilon$. As a matter of fact, $v_{\| i}$ may become negative at the MP entrance when $\theta_{\phi}$ becomes large, as shown later in the kinetic simulations presented in Sec. IV. Finally, we note that in Ref. 19 the case $\theta_{\phi} \neq 0$ was studied, neglecting the fourth term on the left hand side of Eq. (3). This leads to a different expression for $v_{\| i}$ than in Eq. (17).

\section{B. Density and potential}

The density and potential gradients at the MP entrance can be obtained by observing that, being $\operatorname{det}(\mathbf{M})=0$, the system $\mathbf{M} \vec{X}=0$ allows us to relate among themselves the components of $\vec{X}$, namely the gradients of $n, v_{\| i}$ and $\phi$ in the $s$ direction. In particular, we choose to express $\partial_{s} \phi$ and $\partial_{s} n$ as a function of $\partial_{s} v_{\| i}$. The second and third equations of the system $\mathbf{M} \vec{X}=0$ provide

$$
\begin{gathered}
\partial_{s} \phi=-\frac{v_{s i}}{\sin \alpha-\cos \alpha \partial_{x} v_{\| i}} \partial_{s} v_{\| i} \\
\partial_{s} n=\frac{n}{T_{e}} \partial_{s} \phi .
\end{gathered}
$$

Using Eq. (16) to express $v_{s i}$ and again retaining only first order terms in $\theta_{n}$ and $\theta_{T_{e}}$, we obtain

$$
\begin{gathered}
\partial_{s} \phi=-c_{s}\left(1+\theta_{n}+\theta_{T_{e}} / 2\right) \partial_{s} v_{\| i}, \\
\partial_{s} n=-\left(n / c_{s}\right)\left(1+\theta_{n}+\theta_{T_{e}} / 2\right) \partial_{s} v_{\| i} .
\end{gathered}
$$

\section{Temperature}

The MP entrance condition was derived assuming no temperature gradient in the $s$ direction. For consistency,

$$
\partial_{s} T_{e}=0,
$$

can be used as a boundary condition for the electron temperature. A more detailed calculation that takes into account temperature variations is presented in the Appendix and shows that the temperature gradient at the MP entrance is indeed small.

\section{Vorticity}

The vorticity represents the curl of the $\mathbf{E} \times \mathbf{B}$ drift in the parallel direction, being defined as $\omega=[\nabla \times(\mathbf{E} \times \mathbf{b})] \cdot \mathbf{b}$ $=\nabla_{\perp}^{2} \phi$, and it measures the frequency of the plasma rotation in the plane perpendicular to the magnetic field. In the system under consideration, we can write $\nabla^{2} \phi=\partial_{x}^{2} \phi$ $+\partial_{y}^{2} \phi=\partial_{y}^{2} \phi+O\left(\epsilon^{2}\right)$, and the term of order $\epsilon^{2}$ can be neglected. Moreover, we have $\partial_{y}^{2} \phi=\cos ^{2} \alpha \partial_{s}^{2} \phi$, and $\partial_{s}^{2} \phi$ at the MP entrance can be estimated by computing the derivative of Eq. (19) along the $s$ direction and then using again Eq. (16) to express $v_{s i}$. This leads to

$$
\omega=-\cos ^{2} \alpha\left[\left(1+\theta_{T_{e}}\right)\left(\partial_{s} v_{\| i}\right)^{2}+c_{s}\left(1+\theta_{n}+\theta_{T_{e}} / 2\right) \partial_{s}^{2} v_{\| i}\right] .
$$

\section{E. Parallel electron velocity}

While in the MP electrons are always magnetized since $\rho_{e} \ll \rho_{s}$, in the DS the electron dynamics depends on the relative magnitude between $\lambda_{D}$ and $\rho_{e}$. We focus on the $\rho_{e} \ll$ $\lambda_{D}$ regime, where electrons remain magnetized all the way up to the wall, and the value of $v_{\| e}$ at the MP entrance essentially depends on $\eta_{m}=\left(\phi_{M P E}-\phi_{W}\right) / T_{e}$, the normalized potential drop from the MP entrance to the wall. A detailed kinetic treatment of the electron trajectories, taking into account the presence of gradients in the $x$ direction, leads to the following result ${ }^{15}$ :

$$
v_{\| e}=c_{s} \exp \left(\Lambda-\eta_{m}\right)-\frac{\partial_{x} \phi}{\tan \alpha}+\frac{\partial_{x} p_{e}}{n \tan \alpha},
$$

where $\Lambda=\log \sqrt{\mu / 2 \pi}$, and $\partial_{x} p_{e} / n$ is the diamagnetic drift velocity. Using the definition of $\theta_{n}, \theta_{T_{e}}$ and $\theta_{\phi}$, Eqs. (14), (15), and (18), we can write Eq. (25) as

$$
v_{\| e}=c_{s}\left[\exp \left(\Lambda-\eta_{m}\right)-\frac{2 \phi}{T_{e}} \theta_{\phi}+2\left(\theta_{n}+\theta_{T_{e}}\right)\right],
$$

which shows that both potential and diamagnetic corrections are comparable. We remark that if $\rho_{e} \gtrsim \lambda_{D}$, electron trajectories may become rather complex in the Debye sheath, ${ }^{23,24}$ and it is not possible to find a simple expression for $v_{\| e}$ as in Eq. (25).

Equation (26) together with Eqs. (17), (21)-(24), constitute the boundary conditions to be implemented in plasma fluid turbulence codes at the MP entrance.

\section{PARTICLE SIMULATIONS OF THE MAGNETIC PRESHEATH}

In order to confirm the validity of the analytical results presented in Secs. II and III, we perform numerical simulations with the ODISEE (One-DImensional Sheath Edge Explorer) code, ${ }^{22,26}$ a fully kinetic, electrostatic particle-incell (PIC) code akin to previous simulations, ${ }^{27,28}$ which solves the Vlasov-Poisson system in one dimension in real space and three dimensions in velocity space. ODISEE is used to simulate a one-dimensional plasma bound between two absorbing walls at $s=0$ and $s=L$, being the size of the system much larger than the sheath scale, i.e., $L \simeq 20 \rho_{s} \simeq 10^{3} \lambda_{D}$. A source of ions and electrons uniformly distributed in space maintains the plasma in steady state. Ions have a temperature much smaller than the electrons, $T_{i} / T_{e} \simeq 10^{-2}$, and electrons undergo elastic collisions with each other according to a Fokker-Plank collision operator, ${ }^{29}$ 
with a mean free path $\lambda_{m f p}$ smaller than the system size but much larger than the sheath scale, $L>\lambda_{m f p} \gg \lambda_{D}$. As a consequence, electrons present a thermalized distribution function far from the walls, while the sheath remains essentially collisionless. Poisson's equation is solved by imposing a fixed potential $\phi_{w}=0$ at the two boundaries, and the magnetic field is constant and tilted with respect to the wall at an angle $\alpha$, as shown in Fig. 1. Parameters are chosen such that the scaling $\rho_{e} \lesssim \lambda_{D} \ll \rho_{s} \ll \lambda_{m f p}<L$ is ensured. In particular, to guarantee, $\rho_{s} / \rho_{e}=\sqrt{\mu} \gg 1$, the realistic mass ratio $\mu=1836$ is used in the simulations. After a transient phase, a quasi-steady

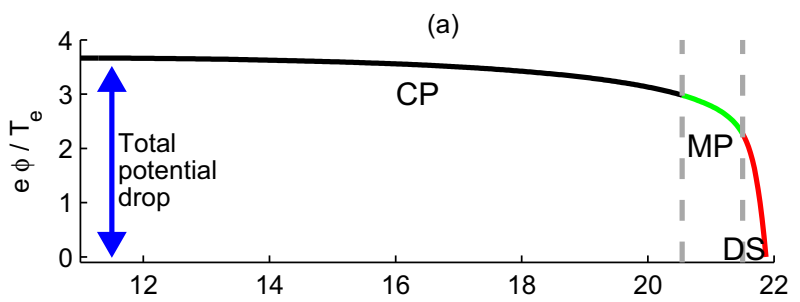

(b)
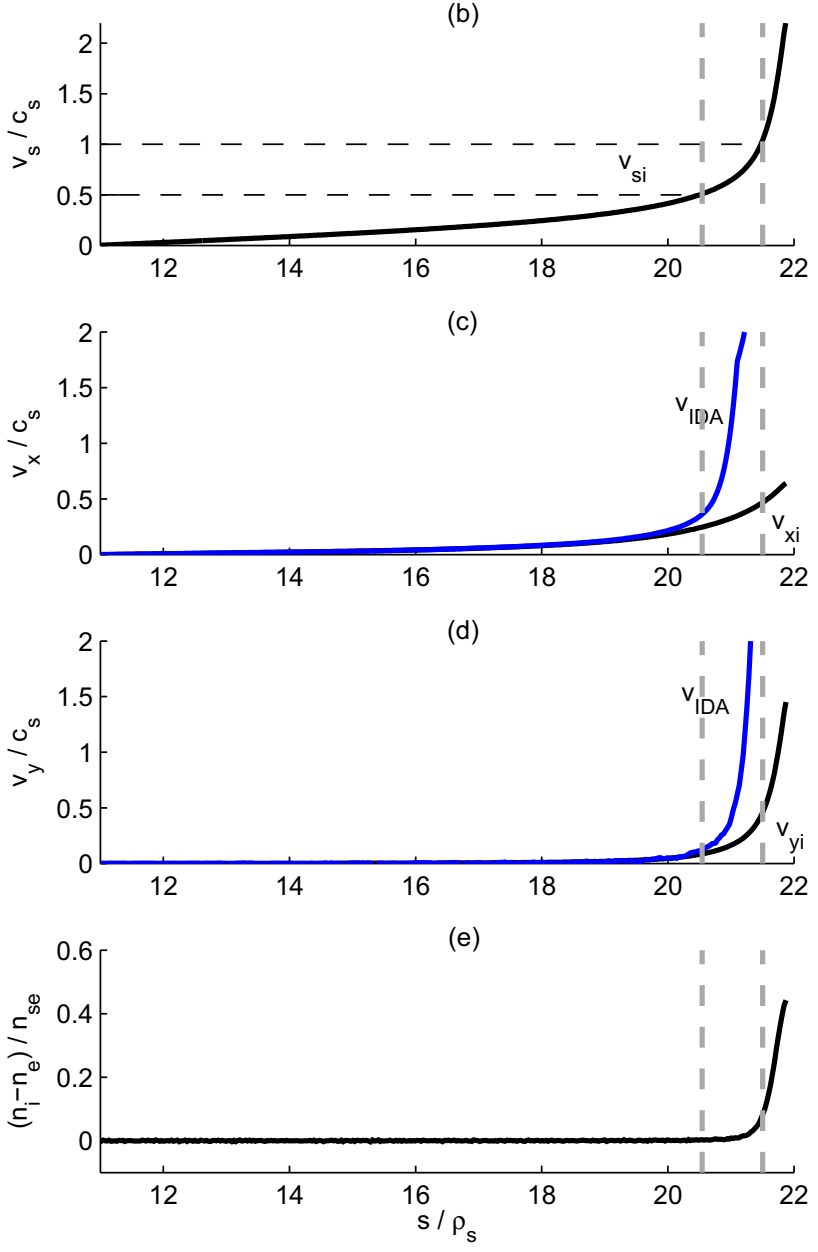

FIG. 2. Time-averaged profiles in proximity of the $s=L$ wall, obtained from the PIC simulations described in Sec. IV, with $\alpha=30^{\circ}$ : (a) electrostatic potential, (b) ion velocity in the $s$ direction, (c) ion velocity in the $x$ direction (bottom, black) and the corresponding velocity as given by the IDA (top, blue), in this case $v_{x i}=-\cos \alpha \partial_{s} \phi$ according to Eq. (2), (d) ion velocity in the $y$ direction (bottom, black) and the corresponding velocity as given by the IDA (top, blue), in this case $v_{y i}=-v_{\| i} \sin \alpha \cos \alpha \partial_{s}^{2} \phi$ according to Eq. (2), (e) charge imbalance normalized to the density at the DS entrance. Vertical dashed lines indicate the location of the MP entrance and the DS entrance. Horizontal dashed lines indicate Mach numbers $M=1$ and $M=\sin \alpha=0.5$. state is achieved in the system, which is a balance between the plasma sources and the losses at the walls. The results presented here focus on this quasi-steady state. In particular, we first show that the main features of the CP, MP, and DS described in Sec. II are retrieved by the simulations, and then we verify the boundary conditions presented in Sec. III.

In order to describe the main features of the plasma-wall transition, we start by considering floating conditions, namely no net current to the walls, and no gradients in the $x$ direction, i.e. $\theta_{n}=\theta_{T_{e}}=\theta_{\phi}=0$. This is achieved by setting equal ion and electron particle sources, $S_{p i}=S_{p e}$. Figures 2(a) and 2(b) show the time-averaged profiles of the plasma potential and the ion velocity $v_{s i}$, in proximity of the $s=L$ wall (exactly the same consideration can be made for the $s=$ 0 wall). In the CP (black region in Fig. 2(a)), ions are accelerated and, according to the analytical derivation of Sec. II, the entrance of the MP is defined by the point where $v_{s i}=c_{s} \sin \alpha$, in correspondence of which the IDA is expected to break down. This is confirmed by Figs. 2(c) and 2(d), where one observes that in the MP the ion perpendicular dynamics can no longer be described by the IDA, Eq. (2). In Fig. 2(a), one can see that the thickness of the MP (green region in Fig. 2(a)) is of the order of $\rho_{s}$. In this region, ions are accelerated from $v_{s i}=c_{s} \sin \alpha$ to $v_{s i}=c_{s}$, as evident from Fig. 2(b). The entrance of the DS corresponds to the point where ions reach the sound speed along the $s$ direction, $v_{s i}=c_{s}$, and inside the DS (red region in Fig. 2(a)) quasineutrality is violated, as visible in Fig. 2(e).

The results shown in Fig. 2 are all relative to the $\alpha=$ $30^{\circ}$ case; the effect of the angle $\alpha$ on the plasma potential in the different regions of the plasma-wall transition remains to be discussed. Since in the MP ions are accelerated from $v_{s i}=c_{s} \sin \alpha$ to $v_{s i}=c_{s}$, the potential drop from the MP entrance to the DS entrance, $\Delta \phi_{M P}$, depends on $\alpha$. We can estimate $\Delta \phi_{M P}$ by observing that the ion flux is approximately constant throughout the sheath. Therefore the ratio of the ion velocities at the DS entrance and at the MP entrance is

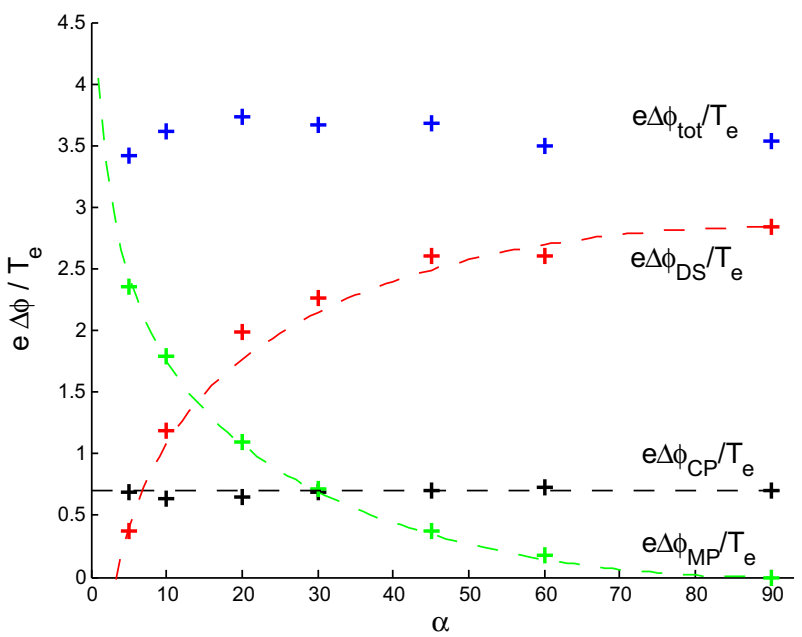

FIG. 3. Potential drop in the CP (black crosses), MP (green crosses), DS (red crosses), for different values of $\alpha$. Results are obtained from the PIC simulations described in Sec. IV. The total potential drop is also indicated (blue crosses). The horizontal black dashed line indicates the value $e \Delta \phi / T_{e}=0.7$, while the green dashed line represents the function $e \Delta \phi / T_{e}=-\log (\sin \alpha)$ and the red dashed line is $e \Delta \phi / T_{e}=\Lambda+\log (\sin \alpha)$. 
inversely proportional to the ratio of densities at the same locations. Assuming that the ratio of densities is given by the Boltzmann factor, we deduce $e \Delta \phi_{M P} / T_{e}=-\log (\sin \alpha)$, as confirmed by Fig. 3. On the other hand, $\Delta \phi_{D S}$, the potential drop in the DS, has the opposite trend (see Fig. 3), in such a way that the total drop from the MP entrance to the wall is always equal to the floating potential, $\eta_{m}=\Lambda$. Finally, $\Delta \phi_{C P}$, the potential drop in the CP, depends on the specific presheath process present in the plasma, such as collisions or sources. Since those are independent of $\alpha$ in our simulations, $\Delta \phi_{C P}$ does not depend on $\alpha$, as shown in Fig. 3, being $e \Delta \phi_{C P} / T_{e} \approx 0.7$, as predicted in Ref. 25 .

Turning now to the validity of the boundary conditions derived in Sec. III, we note that a constant electric field $E_{x}$ can be included in the one-dimensional model considered by ODISEE, whereas plasma scenarios with $\theta_{n} \neq 0$ and $\theta_{T_{e}} \neq 0$ cannot be simulated. We thus limit ourselves to the analysis of the finite $E_{x}$ effect, which corresponds to a finite $\theta_{\phi}$ in the

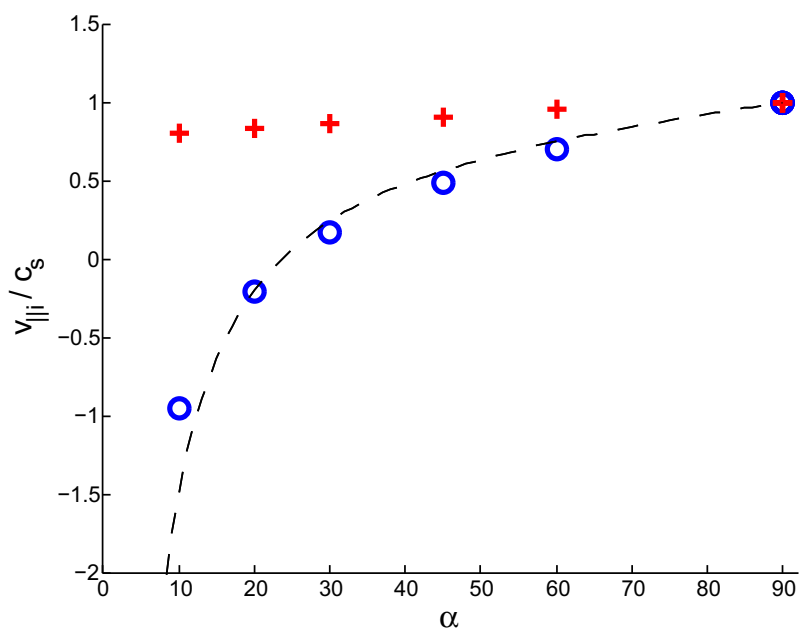

(a)

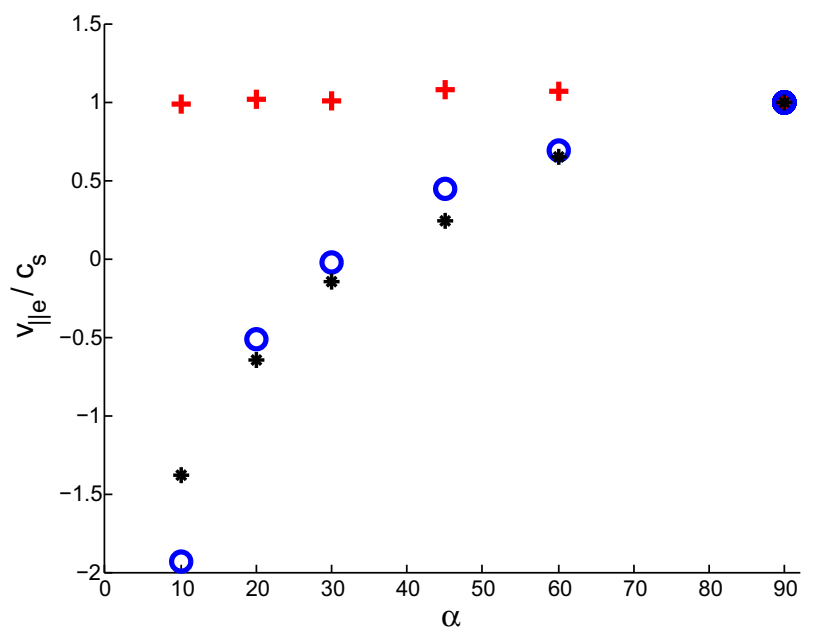

(b)

FIG. 4. Ion (top) and electron (bottom) parallel velocities at the MP entrance as a function of $\alpha$, for $E_{x}=0$ (red crosses) and $E_{x} / B=-0.2 c_{s}$ (blue circles). Results are obtained from the PIC simulations described in Sec. IV. Dashed line on the top panel is the function $f(\alpha)=1-0.2 / \tan \alpha$, which denotes the expected value from the proposed set of boundary conditions. Black stars on the bottom panel denote $g(\alpha)=\exp \left(\Lambda-\eta_{m}\right)-0.2 / \tan \alpha$, the expected value, being $\eta_{m}$ the potential barrier at the MP entrance observed in the simulations for each value of $\alpha$. boundary conditions. Figure 4 shows that the ion parallel velocity at the MP entrance is, within a good approximation, independent of $\alpha$ when $E_{x}=0$, while it follows rather well Eq. (17) when $E_{x} \neq 0$. We note that the small discrepancy observed in the $E_{x}=0$ case with respect to Eq. (17), $v_{\| i}=c_{s}$, is due to the contribution of the polarization drift (see Fig. 2(d)) which is not taken into account in the derivation of the boundary conditions. For the case $E_{x} \neq 0$, it is interesting to notice that, for sufficiently large $\mathbf{E} \times \mathbf{B}$ correction, one has $v_{\| i}<0$, thus indicating that particles are flowing, in the parallel direction, from the wall into the main plasma. As a matter of fact, the flow in the direction normal to the wall is given by the MP entrance condition, $v_{s i}=c_{s} \sin \alpha$, which is independent of $E_{x}$. Since $v_{s i}=v_{\| i} \sin \alpha+v_{y i} \cos \alpha$, particles that are convected in the $y$ direction at the $\mathbf{E} \times \mathbf{B}$ velocity must travel backwards, into the main plasma, along the magnetic field in order to ensure that $v_{s i}=c_{s} \sin \alpha$, from which $v_{\| i}<0$.

The electron parallel velocity at the MP entrance is also shown in Fig. 4 as a function of $\alpha$, showing good agreement with Eq. (26) in both the $E_{x}=0$ and the $E_{x} \neq 0$ cases. In order to verify the dependence of the boundary condition for the parallel electron velocity on the potential barrier $\eta_{m}$, which in the limit $\theta_{n}=\theta_{T_{e}}=\theta_{\phi}=0$ is $v_{\| e}=c_{s} \exp \left(\Lambda-\eta_{m}\right)$, we explore the steady-state of the system in non-ambipolar conditions. A non-neutral particle source is considered, $S_{p i} \neq S_{p e}$, inducing a net current to the walls and therefore modifying the value of the potential at the MP entrance. Figure 5 shows the value of $v_{\| e}$ at the MP entrance, as a function of the potential at this same position. The results are compared with the analytical prediction, showing a fairly good agreement. Moreover Fig. 5 shows that $v_{\| i}$ at the MP entrance is independent of $\eta_{m}$.

Finally, we verify the expressions for the gradients of potential and density at the MP entrance, Eqs. (21) and (22), and for the vorticity, Eq. (24), by comparing those with the numerical values from the simulations. This is shown in Fig. 6 . The agreement is very good for the potential gradient and

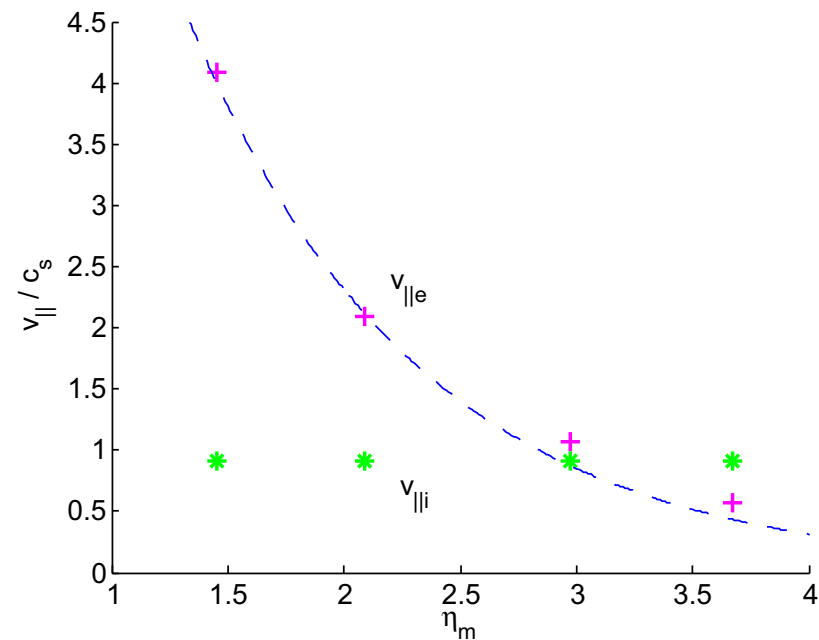

FIG. 5. Ion (green stars) and electron (magenta crosses) parallel velocities at the MP entrance as a function of $\eta_{m}$, for $\alpha=45^{\circ}$. Results are obtained from the PIC simulations described in Sec. IV. The dashed curve represents the function $\exp \left(\Lambda-\eta_{m}\right)$. 


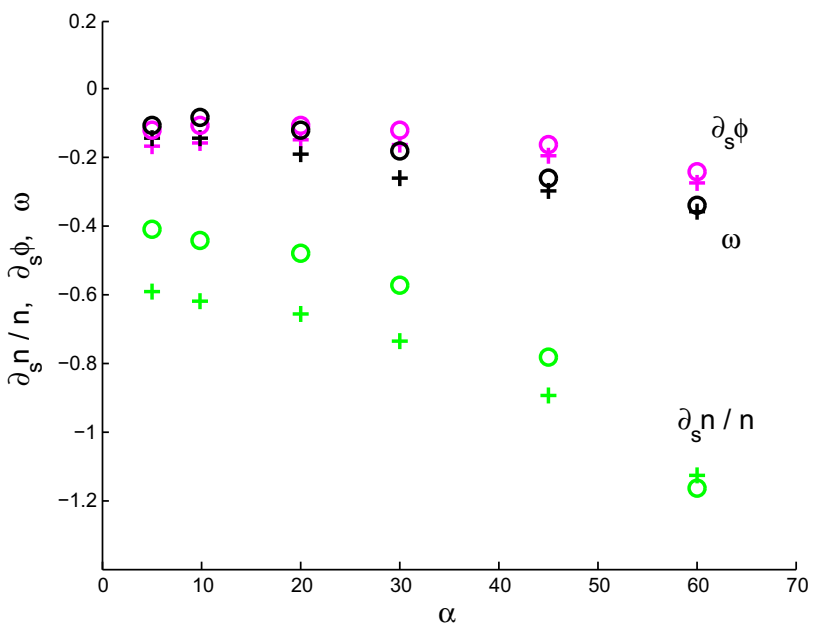

FIG. 6. Comparison between PIC simulation results (crosses) and the boundary conditions (circles), expressed in Eqs. (21), (22), and (24), for the potential gradient $\partial_{s} \phi$ (magenta), the density gradient $\partial_{s} n / n$ (green), and the vorticity $\omega$ (black), at the MP entrance. for the vorticity, and reasonable for the density gradient. The difference between simulation results and the analytical expressions is mainly due to the effect of $S_{p i} \neq 0$ and $S_{p e} \neq$ 0 in the MP.

\section{FLUID SIMULATIONS WITH BOUNDARY CONDITIONS AT THE MAGNETIC PRESHEATH ENTRANCE}

We have implemented the set of boundary conditions for the MP entrance, Eqs. (17), (21), (22), (23), (24), and (26), in the GBS code, ${ }^{6}$ a global three-dimensional fluid code based on the drift-reduced Braginskii equations with $T_{i} \ll T_{e}$ and the Boussinesq approximation. GBS evolves the plasma dynamics with no separation between equilibrium and fluctuating quantities, as a balance between density and heat sources, the turbulent cross-field transport produced by plasma instabilities, and the losses at the sheaths, where the magnetic field lines terminate on the walls. GBS has been used to simulate linear devices such as LAPD,${ }^{30}$ simple magnetized
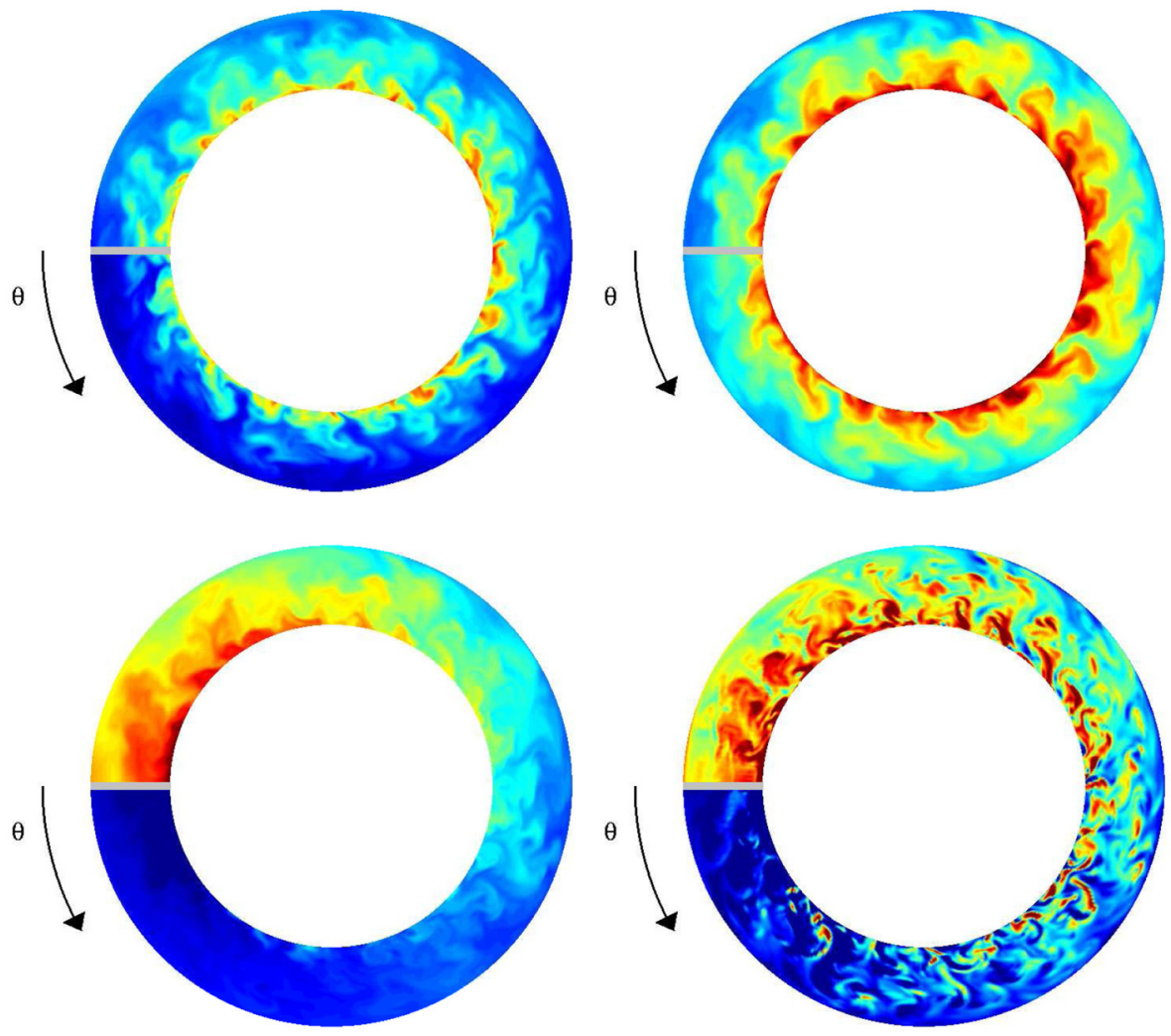

FIG. 7. Snapshots in a poloidal cross-section of the density (top left), temperature (top right), parallel ion (middle left) and electron (middle right) velocities, electrostatic potential (bottom left) and vorticity (bottom right). Results are obtained from the GBS simulations of a limited tokamak SOL, as described in Sec. V. Boundary conditions at the MP entrance are implemented at the limiter plate, located at $\theta=0$. The snaphots cover the radial extension $\left(r_{s}, r_{\max }\right)$.
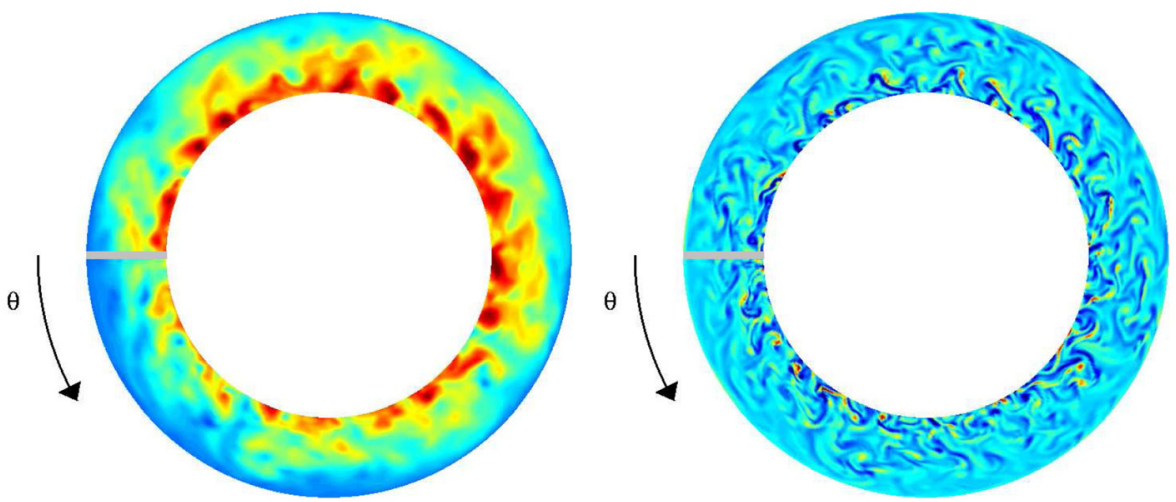
toroidal devices such as TORPEX, ${ }^{31}$ and the tokamak SOL. ${ }^{6}$ GBS results have been fully validated against experimental measurements. ${ }^{32,33}$ The system of equations evolved by GBS is, in the electrostatic limit,

$$
\begin{aligned}
\frac{\partial n}{\partial t}= & -\frac{R}{\rho_{s 0}} \frac{1}{B}[\phi, n]+\frac{2 n}{B}\left[C\left(T_{e}\right)+\frac{T_{e}}{n} C(n)-C(\phi)\right] \\
& -n(\mathbf{b} \cdot \nabla) V_{\| e}-V_{\| e}(\mathbf{b} \cdot \nabla) n+\mathcal{D}_{n}(n)+S \\
\frac{\partial \omega}{\partial t}= & -\frac{R}{\rho_{s 0}} \frac{1}{B}[\phi, \omega]-V_{\| i}(\mathbf{b} \cdot \nabla) \omega+B^{2}\left[(\mathbf{b} \cdot \nabla)\left(V_{\| i}-V_{\| e}\right)\right. \\
+ & \left.\frac{\left(V_{\| i}-V_{\| e}\right)}{n}(\mathbf{b} \cdot \nabla) n\right]+2 B\left[C\left(T_{e}\right)+\frac{T_{e}}{n} C(n)\right] \\
+ & \frac{B}{3 n} C\left(G_{i}\right)+\mathcal{D}_{\omega}(\omega), \\
\frac{\partial V_{\| e}}{\partial t}= & -\frac{R}{\rho_{s 0}} \frac{1}{B}\left[\phi, V_{\| e}\right]-V_{\| e}(\mathbf{b} \cdot \nabla) V_{\| e}-\frac{m_{i}}{m_{e}} \frac{2}{3}(\mathbf{b} \cdot \nabla) G_{e} \\
& -\frac{m_{i}}{m_{e}} \nu\left(V_{\| e}-V_{\| i}\right)+\frac{m_{i}}{m_{e}}(\mathbf{b} \cdot \nabla) \phi-\frac{m_{i} T_{e}}{n m_{e}}(\mathbf{b} \cdot \nabla) n \\
& -1.71 \frac{m_{i}}{m_{e}}(\mathbf{b} \cdot \nabla) T_{e}+\mathcal{D}_{V_{\| e}}\left(V_{\| e}\right),
\end{aligned}
$$

$$
\begin{aligned}
\frac{\partial V_{\| i}}{\partial t}= & -\frac{R}{\rho_{s 0}} \frac{1}{B}\left[\phi, V_{\| i}\right]-V_{\| i}(\mathbf{b} \cdot \nabla) V_{\| i}-\frac{2}{3}(\mathbf{b} \cdot \nabla) G_{i} \\
& -\left[(\mathbf{b} \cdot \nabla) T_{e}+\frac{T_{e}}{n}(\mathbf{b} \cdot \nabla) n\right]+\mathcal{D}_{V_{\| i}}\left(V_{\| i}\right),
\end{aligned}
$$

$$
\begin{aligned}
\frac{\partial T_{e}}{\partial t}= & -\frac{R}{\rho_{s 0}} \frac{1}{B}\left[\phi, T_{e}\right]-V_{\| e}(\mathbf{b} \cdot \nabla) T_{e} \\
& +\frac{4}{3} \frac{T_{e}}{B}\left[\frac{7}{2} C\left(T_{e}\right)+\frac{T_{e}}{n} C(n)-C(\phi)\right] \\
& +\frac{2 T_{e}}{3}\left[0.71(\mathbf{b} \cdot \nabla) V_{\| i}-1.71(\mathbf{b} \cdot \nabla) V_{\| e}\right. \\
& \left.+0.71 \frac{\left(V_{\| i}-V_{\| e}\right)}{n}(\mathbf{b} \cdot \nabla) n\right] \\
& +\mathcal{D}_{T_{e}}\left(T_{e}\right)+\mathcal{D}_{T_{e}}^{\|}\left(T_{e}\right)+S_{T},
\end{aligned}
$$

which are coupled to the Poisson equation $\nabla_{\perp}^{2} \phi=\omega$. Quantities are normalized as described in Sec. II, except for the parallel direction which is normalized to a macroscopic scale length $R$ instead of $\rho_{s 0}$. The resistivity $\nu$ is normalized to $c_{s 0} / R$ and the magnetic field $B$ is normalized to the reference magnetic field $B_{0}$. The curvature operator is defined as

$$
C(f) \equiv \frac{B}{2}\left(\nabla \times \frac{\mathbf{b}}{B}\right) \cdot \nabla f
$$

and $[f, g]=\mathbf{b} \cdot(\nabla f \times \nabla g)$. The diffusion operators $\mathcal{D}$ and $\mathcal{D}^{\|}$are introduced for numerical purposes, and the gyroviscous terms are denoted by $G_{i}$ and $G_{e}$ (see Ref. 6 for an explicit definition). We remark that similar sets of equations, based on the drift-reduced approach, ${ }^{34}$ are considered by a number of other codes (see, e.g., Refs. 2-8).

We have implemented in GBS the boundary conditions at the MP entrance for $n, \omega, V_{\| i}, V_{\| e}, T_{e}$ and $\phi$, according to
Eqs. (17), (21), (22), (23), (24), and (26), using a second order finite difference scheme. Since in GBS we typically consider situations where the angle between the magnetic field and the wall is very small, $\alpha \ll 1$, it is possible to assume that $\partial_{s}=\partial_{y}$ in the expressions of the boundary conditions, thus simplifying the numerical implementation. According to the hypothesis of a sheath in steady-state conditions and with $\epsilon \ll 1$, the terms involving finite radial gradients corrections, $\theta_{n}, \theta_{T_{e}}$, and $\theta_{\phi}$, are time-averaged over a time window of the order of $1 R / c_{s 0}$, and are spatially smoothed in the $x$ direction by suppressing oscillations having length scales shorter than $10 \rho_{s 0}$.

We focus here on tokamak SOL simulations with circular magnetic flux surfaces, no magnetic shear, and a toroidal limiter located on the equatorial plane, at the high-field side. We denote with $(x, y)$ the coordinates perpendicular to the magnetic field, $x$ being the radial coordinate and $y$ being perpendicular to both $x$ and $\mathbf{b}$, thus corresponding approximately to the poloidal coordinate. In this geometry we have $C=$ $-\sin (y / 2 \pi a) \partial_{x} \quad-\cos (y / 2 \pi a) \partial_{y},[f, g]=\partial_{x} g \partial_{y} f-\partial_{x} f \partial_{y} g$, and $\nabla_{\perp}^{2}=\partial_{x}^{2}+\partial_{y}^{2}$. Here $a$ is the tokamak minor radius. We use the following model parameters: major radius $R=500 \rho_{s 0}$, aspect ratio $a / R \approx 0.25$, radial extension $L_{x}=r_{\max }-r_{\min }=100 \rho_{s 0}$, safety factor $q=4$, and resistivity $\nu=0.1$. The angle between the magnetic field and the limiter is such that $\tan \alpha=a / q R \approx 0.0625$, corresponding to $\alpha \approx 3.6^{\circ}$. The particle and heat outflow from the core is modeled by density and temperature Gaussian sources that are radially-localized at $r=r_{s}=r_{\min }+30 \rho_{s 0}$. We apply the MP boundary conditions at the limiter plates, i.e., at $y=0$ and $y=2 \pi a$, and we note that Neumann boundary conditions are imposed at $r_{\min }$ and $r_{\max }$ for all fields, except for $\phi$, which is set to $\phi=\Lambda T_{e}$.

Figure 7 shows snapshots of the different fields in a poloidal cross-section of the torus. A detailed analysis of the simulation results will be the subject of future publications. Here we note, from a qualitative point of view, that smooth
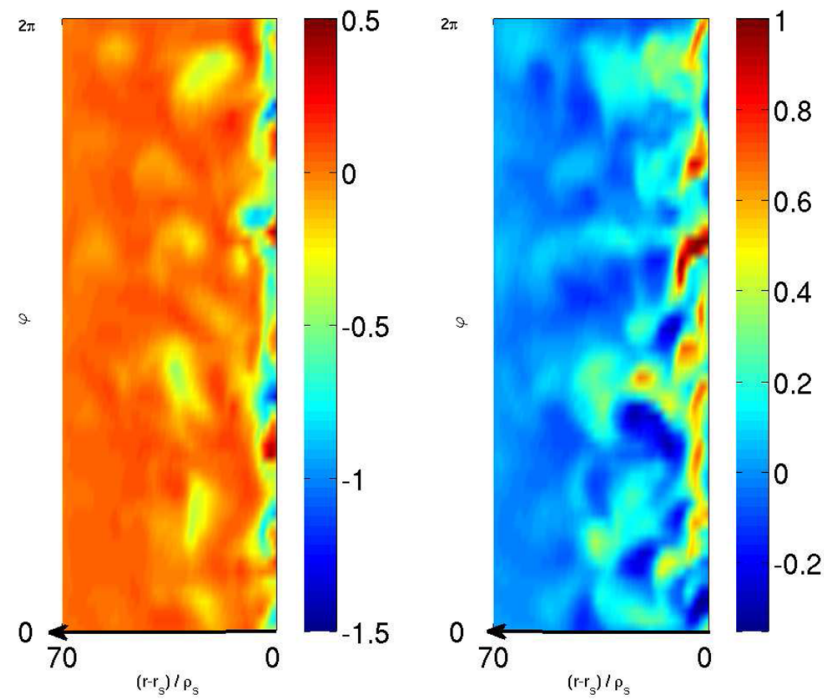

FIG. 8. Snapshot of parallel currents $j_{\|}=e n\left(v_{\| i}-v_{\| e}\right)$ flowing to the top (left panel) and bottom (right panel) sides of the limiter. The coordinate $\varphi$ denotes the toroidal angle. Results are obtained from the GBS simulations described in Sec. V. 
profiles form at the limiters, suggesting that the set of boundary conditions for the plasma-wall transition is compatible with the GBS model equations. Also, the plasma potential can fluctuate at the limiter and thus allows for finite parallel currents, as typically observed at the edge of basic plasma physics experiments $^{35}$ and at the limiters or divertors of tokamaks. ${ }^{36}$ Figure 8 shows a snapshot of the parallel currents that form at both sides of the limiter.

\section{CONCLUSIONS}

We have provided a complete set of analytical boundary conditions at the MP entrance for plasma fluid turbulence codes based on the IDA. These are summarized below for convenience, for both sides of the field line,

$$
\begin{gathered}
v_{\| i}=c_{s}\left( \pm 1+\theta_{n} \mp \frac{1}{2} \theta_{T_{e}}-\frac{2 \phi}{T_{e}} \theta_{\phi}\right) \\
v_{\| e}=c_{s}\left( \pm \exp \left(\Lambda-\eta_{m}\right)-\frac{2 \phi}{T_{e}} \theta_{\phi}+2\left(\theta_{n}+\theta_{T_{e}}\right)\right) \\
\partial_{s} \phi=-c_{s}\left( \pm 1+\theta_{n} \pm \frac{1}{2} \theta_{T_{e}}\right) \partial_{s} v_{\| i}, \\
\partial_{s} n=-\frac{n}{c_{s}}\left( \pm 1+\theta_{n} \pm \frac{1}{2} \theta_{T_{e}}\right) \partial_{s} v_{\| i} \\
\partial_{s} T_{e}=0
\end{gathered}
$$

$\omega=-\cos ^{2} \alpha\left[\left(1+\theta_{T_{e}}\right)\left(\partial_{s} v_{\| i}\right)^{2}+c_{s}\left( \pm 1+\theta_{n} \pm \theta_{T_{e}} / 2\right) \partial_{s}^{2} v_{\| i}\right]$,

where the upper signs apply if the magnetic field is directed towards the wall, and the lower signs apply in the opposite case. We now make a few comments on the newly derived boundary conditions.

For the parallel ion and electron velocities, Eqs. (33) and (34), the corrections due to $\mathbf{E} \times \mathbf{B}$ and diamagnetic drifts might have a significant impact. In fact, in the presence of strong radial gradients, the parallel velocities may display an inflowing character, as already discussed in Sec. IV and observed in Fig. 4. To our knowledge, while this effect has already been suggested in the literature, ${ }^{25}$ these corrections to the parallel velocities have never been implemented in plasma turbulence codes. The potential gradient in Eq. (35), $\partial_{s} \phi$, is proportional to $\partial_{s} v_{\| i}$. Since ions are accelerated towards the wall, we typically have $\partial_{s} v_{\| i}>0$, and therefore $\partial_{s} \phi<0$, which is consistent with the potential drop expected in the vicinity of the wall. Similarly for the density gradient, Eq. (36): we expect $\partial_{s} n<0$, consistent with the conservation of ion particle flux. The vorticity, Eq. (38), is also expected to be negative, $\omega<0$, setting the direction of rotation of the $\mathbf{E} \times \mathbf{B}$ flow at the edge. This is consistent with the fact that the sheath electric field, which induces an $\mathbf{E} \times \mathbf{B}$ flow parallel to the wall, increases when approaching the MP entrance.

We remark that in the limit of $\alpha \rightarrow \pi / 2$, namely, when the magnetic field is perpendicular to the wall, the MP disappears and the plasma-wall transition region is reduced to the presence of the Debye sheath. In this limit, $\theta_{n}, \theta_{T}, \theta_{\phi} \rightarrow 0$, and the boundary conditions for $V_{\| i}$ and $V_{\| e}$ reduce to the Bohm boundary conditions at the Debye sheath entrance. Finally, we notice that this set of boundary conditions is not limited to 3D fluid models ${ }^{4-6}$ but may also be applied to 2D fluid models, ${ }^{2,3}$ where expressions for $V_{\| i}$ and $V_{\| e}$ are needed at the MP entrance.

With the set of boundary conditions at the MP entrance presented here, it becomes possible to describe the plasma dynamics in an open magnetic field line geometry with a model based on the IDA, still taking into account properly the sheath physics. This set of boundary conditions has been confirmed by PIC simulations of the magnetized plasmawall transition and implemented in the GBS code. Results indicate compatibility with three-dimensional global fluid turbulence simulations.

We finally remark that while this set of boundary conditions faithfully supplies the sheath physics to the fluid codes, it remains simple and easy to implement. Additional effects such as finite ion temperature, finite ion inertia, secondary electron emission, sputtering, magnetic curvature, finite aspect ratio, or electromagnetic effects, can also be included in the boundary conditions within the framework presented here.

\section{ACKNOWLEDGMENTS}

Simulations were performed on the HPC-FF cluster of the Jülich Forschungszentrum. We acknowledge many useful discussions with A. Fasoli, I. Furno, K. Gustafson, C. Theiler, and T.-M. Tran. We also acknowledge financial support by the Fonds National Suisse de la Recherche Scientifique.

\section{APPENDIX: MAGNETIC PRESHEATH ENTRANCE CONDITION WITH NON-ISOTHERMAL ELECTRONS}

Here we present the derivation of the MP entrance condition when the assumption of isothermal electrons is relaxed. For the sake of simplicity, we consider the case of no gradients in the $x$ direction. Therefore, according to the IDA one has $v_{y i}=0$ and thus $v_{s i}=v_{\| i} \sin \alpha$. Considering non-isothermal electrons requires the use of a heat equation, such as Eq. (31), which in steady-state conditions and neglecting inertia, diffusion and resistivity effects, can be written as

$$
\begin{aligned}
n v_{\| e} \sin \alpha \partial_{s} T_{e} & +\frac{2 T_{e}}{3}\left[1.71 n \sin \alpha \partial_{s} v_{\| e}-0.71 n \sin \alpha \partial_{s} v_{\| i}\right. \\
& \left.-0.71\left(v_{\| i}-v_{\| e}\right) \sin \alpha \partial_{s} n\right]=S_{T} .
\end{aligned}
$$

For non-isothermal electrons, the parallel electron momentum equation, Eq. (7), gives

$$
\mu \sin \alpha T_{e} \partial_{s} n+1.71 \mu n \sin \alpha \partial_{s} T_{e}-\mu n \sin \alpha \partial_{s} \phi=S_{\| m e},
$$

where we have included the contribution of the thermal force, $0.71 \mu n \sin \alpha \partial_{s} T_{e}$, in GBS Ohm's law, Eq. (29).

We assume that the parallel electron velocity can be expressed as $v_{\| e}=v_{\| e}\left(\phi, T_{e}\right)$ in the proximity of the MP entrance. It follows that $\partial_{s} v_{\| e}=c_{\phi} \partial_{s} \phi+c_{T_{e}} \partial_{s} T_{e}$ where $c_{\phi}=$ 
$\partial_{\phi} v_{\| e}$ and $c_{T_{e}}=\partial_{T_{e}} v_{\| e}$ are assumed to be known functions. Equation (A1) can then be written as a linear combination of $\partial_{s} n, \partial_{s} v_{\| i}, \partial_{s} \phi$, and $\partial_{s} T_{e}$. Equations (4), (6), (A2), and (A1) describe the plasma dynamics in the $\mathrm{CP}$ and they can be written as a matrix system, $\mathbf{M} \vec{X}=\vec{S}$, where $\vec{X}=$ $\left(\partial_{s} n, \partial_{s} v_{\| i}, \partial_{s} \phi, \partial_{s} T_{e}\right)$ and $\vec{S}=\left(S_{p i}, S_{\| i}, S_{\| e}, S_{T}\right)$, with

$$
\mathbf{M}=\left(\begin{array}{cccc}
v_{s i} & n \sin \alpha & 0 & 0 \\
0 & n v_{s i} & n \sin \alpha & 0 \\
\mu \sin \alpha T_{e} & 0 & -\mu n \sin \alpha & 1.71 \mu n \sin \alpha \\
\frac{2}{3} 0.71 T_{e}\left(v_{\| e}-v_{\| i}\right) \sin \alpha & -\frac{2}{3} 0.71 n T_{e} \sin \alpha & \frac{2}{3} 1.71 c_{\phi} n T_{e} \sin \alpha & \frac{2}{3} 1.71 c_{T_{e}} n T_{e} \sin \alpha
\end{array}\right) .
$$

We note that, discarding the last row and column of the matrix $\mathbf{M}$, related to temperature fluctuations, one retrieves the matrix obtained in Eq. (11). The condition defining the MP entrance is obtained by imposing $\operatorname{det}(\mathbf{M})=0$, that is

$$
v_{\| i}=c_{s} \sqrt{\frac{1+\frac{2}{3} 1.71\left(\hat{c}_{T_{e}}-0.71\right)}{1+\frac{2}{3} 1.71\left(\hat{c}_{T_{e}}+1.71 \hat{c}_{\phi}\right)}},
$$

where $\hat{c}_{\phi}=c_{\phi} T_{e} / v_{\| e}, \hat{c}_{T_{e}}=c_{T_{e}} T_{e} / v_{\| e}$, and we have used the relation $v_{s i}=v_{\| i} \sin \alpha$. Analytical progress can be achieved by using Eq. (25), $v_{\| e}=\sqrt{T_{e}} \exp \left(\Lambda-\phi / T_{e}\right)$, which gives $\hat{c}_{\phi}=-1$ and $\hat{c}_{T_{e}}=0.5+\phi / T_{e} \simeq 0.5+\Lambda$. Equation (A4) thus gives $v_{\| i} \approx 1.17 c_{s}$ for $\Lambda=3$. Finally, one can get an expression for the temperature gradient,

$$
\partial_{s} T_{e}=\left[\frac{1+0.71\left(1-T_{e} / v_{\| i}^{2}\right)}{\frac{3}{2}+1.71(0.5+\Lambda)}\right] \partial_{s} \phi \approx 0.15 \partial_{s} \phi
$$

which is an order of magnitude smaller than the gradient of the potential, therefore justifying the assumption $\left(\partial_{s} T_{e}=0\right)$ made in Sec. II.

${ }^{1}$ K.-U. Riemann, J. Phys. D: Appl. Phys. 24, 493 (1991).

${ }^{2}$ D. A. Russell, J. R. Myra, and D. A. D'Ippolito, Phys. Plasmas 16, 122304 (2009).

${ }^{3}$ O. E. Garcia, V. Naulin, A. H. Nielsen, and J. Juul Rasmussen, Phys. Rev. Lett. 92, 165003 (2004).

${ }^{4}$ V. Naulin, T. Windisch, and O. Grulke, Phys. Plasmas 15, 012307 (2008).

${ }^{5}$ B. D. Dudson, M. V. Umansky, X. Q. Xu, P. B. Snyder, and H. R. Wilson, Comput. Phys. Commun. 180, 1467 (2009).

${ }^{6}$ P. Ricci, F. D. Halpern, S. Jolliet, J. Loizu, A. Mosetto, A. Fasoli, I. Furno, and C. Theiler, Plasma Phys. Controlled Fusion 54, 124047 (2012).

${ }^{7} \mathrm{P}$. Tamain, Ph. Ghendrih, E. Tsitrone, V. Grandgirard, X. Garbet, Y. Sarazin, E. Serre, G. Ciraolo, and G. Chiavassa, J. Comput. Phys. 229, 361 (2010).
${ }^{8}$ T. T. Ribeiro and B. Scott, Plasma Phys. Controlled Fusion 47, 1657 (2005).

${ }^{9}$ R. Chodura, Phys. Fluids 25, 1628 (1982).

${ }^{10}$ K.-U. Riemann, Phys. Plasmas 1, 552 (1994).

${ }^{11}$ T. M. G. Zimmermann, M. Coppins, and J. E. Allen, Phys. Plasmas $\mathbf{1 5}$ 072301 (2008)

${ }^{12}$ E. Ahedo and D. Carralero, Phys. Plasmas 16, 043506 (2009).

${ }^{13}$ J. Kovačič, T. Gyergyek, and M. Čerček, Eur. Phys. J. D 54, 383 (2009).

${ }^{14}$ N. S. Krasheninikova, X. Tang, and V. S. Roytershteyn, Phys. Plasmas 17, 057103 (2010).

${ }^{15}$ R. H. Cohen and D. D. Ryutov, Phys. Plasmas 2, 2011 (1995).

${ }^{16}$ P. C. Stangeby and A. V. Chankin, Phys. Plasmas 2, 707 (1995).

${ }^{17}$ P. C. Stangeby, Phys. Plasmas 2, 702 (1995).

${ }^{18}$ I. H. Hutchinson, Phys. Plasmas 3, 6 (1996).

${ }^{19}$ D. Tskhakaya and S. Kuhn, Contrib. Plasma Phys. 42, 302 (2002).

${ }^{20}$ M. Stanojević, J. Duhovnik, N. Jelić, A. Kendl, and S. Kuhn, Plasma Phys. Controlled Fusion 47, 685 (2005).

${ }^{21}$ S. Devaux and G. Manfredi, Phys. Plasmas 13, 083504 (2006).

${ }^{22}$ J. Loizu, P. Ricci, and C. Theiler, Phys. Rev. E 83, 016406 (2011)

${ }^{23}$ R. H. Cohen and D. D. Ryutov, Phys. Plasmas 2, 4118 (1995).

${ }^{24}$ R. H. Cohen and D. D. Ryutov, Phys. Plasmas 5, 808 (1998).

${ }^{25} \mathrm{P}$. Stangeby, The Plasma Boundary of Magnetic Fusion Devices (IOP Publishing, Bristol, 2000).

${ }^{26}$ J. Loizu, J. Dominski, P. Ricci, and C. Theiler, Phys. Plasmas 19, 083507 (2012).

${ }^{27}$ J. R. Procassini, C. K. Birdsall, and E. C. Morse, Phys. Fluids B 2, 12 (1990).

${ }^{28}$ J. R. Procassini and C. K. Birdsall, Phys. Fluids B 3, 8 (1991).

${ }^{29}$ T. Takizuka, J. Comput. Phys. 25, 205 (1977).

${ }^{30}$ B. N. Rogers and P. Ricci, Phys. Rev. Lett. 104, 225002 (2010).

${ }^{31}$ P. Ricci and B. N. Rogers, Phys. Rev. Lett. 104, 145001 (2010).

${ }^{32}$ P. Ricci, C. Theiler, A. Fasoli, I. Furno, B. Labit, S. H. Müller, M. Podestà, and F.M. Poli, Phys. Plasmas 16, 055703 (2009).

${ }^{33}$ P. Ricci, C. Theiler, A. Fasoli, I. Furno, K. Gustafson, D. Iraji, and J. Loizu, Phys. Plasmas 18, 032109 (2011).

${ }^{34}$ A. Zeiler, J. F. Drake, and B. Rogers, Phys. Plasmas 4, 2134 (1997).

${ }^{35}$ I. Furno, C. Theiler, D. Lançon, A. Fasoli, D. Iraji, P. Ricci, M. Spolaore, and N. Vianello, Plasma Phys. Controlled Fusion 53, 124016 (2011)

${ }^{36}$ R. A. Pitts, S. Alberti, P. Blanchard, J. Horacek, H. Reimerdes, and P. C. Stangeby, Nucl. Fusion 43, 1145 (2003) 